\title{
Validation of Snort-spit Saliva in Detecting COVID-19 Using RT-PCR and Rapid Antigen Detection Test
}

\author{
Regina P. Berba, MD, MSc ${ }^{1,2}$ and Charlotte M. Chiong, MD, PhD, $1,2,3$

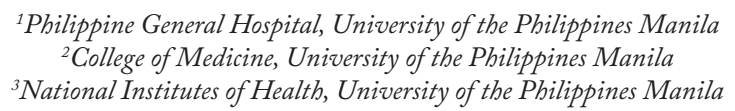

Ryner Jose D. Carrillo, MD, MSc (Clin Epid), ${ }^{* 1,2}$ Abigail D. Sarmiento, MD, ${ }^{* 1}$ Mark Anthony C. Ang, MD, MoS, $, 1,2$

Michele H. Diwa, MD, ${ }^{1,2}$ Cecile C. Dungog, MD, PhD, ${ }^{1}$ Daniel I. Tan, MD,${ }^{1}$ Jan Alexis C. Lacuata, MD, ${ }^{1}$

Jacob Ephraim D. Salud, MD, ${ }^{1}$ Ramon Antonio B. Lopa, MD, ${ }^{1,2}$ John Mark S. Velasco, MD, MPH, MSc, DTM\&H, ${ }^{3}$

\begin{abstract}
Objective. To determine the diagnostic accuracy of self-collected snorted and spit saliva in detecting COVID-19 using RT-PCR (ssRT-PCR) and lateral flow antigen test (ssLFA) versus nasopharyngeal swab RT-PCR (npRT-PCR).
\end{abstract}

Methods. One hundred ninety-seven symptomatic subjects for COVID-19 testing in a tertiary hospital underwent snort-spit saliva self-collection for RT-PCR and antigen testing and nasopharyngeal swab for RT-PCR as reference. Positivity rates, agreement, sensitivity, specificity, and likelihood ratios were estimated.

Results. Estimated prevalence of COVID-19 using npRT-PCR was 9\% (exact 95\% Cl of 5.5\% - 14.1\%). A higher positivity rate of $13 \%$ in the ssRT-PCR assay suggested possible higher viral RNA in the snort-spit samples. There was 92.9\% agreement between ssRT-PCR and npRT-PCR (exact $95 \% \mathrm{Cl}$ of $88.4 \%$ to $96.1 \%$; Cohen's Kappa of 0.6435). If npRT-PCR will be assumed as reference standard, the estimated Sensitivity was $83.3 \%$ (exact $95 \% \mathrm{Cl}$ of $60.8 \%$ to $94.2 \%$ ), Specificity $93.9 \%$ (exact $95 \% \mathrm{Cl}$ of $89.3 \%$ to $96.5 \%$ ), Positive predictive value of $57.7 \%$ (exact $95 \% \mathrm{Cl}$ of $38.9 \%$ to $74.5 \%$ ), Negative predictive value of $98.2 \%$ (exact $95 \% \mathrm{Cl}$ of $95 \%$ to $99.4 \%$ ), positive likelihood ratio of 3.65 (95\% Cl of 7.37 to 24.9$)$, negative likelihood ratio of 0.178 (95\% Cl of 0.063 to 0.499$)$. There was $84.84 \%$ agreement (95\% exact $\mathrm{Cl}$ of $79.1 \%$ to $89.5 \%$; Cohen's Kappa of 0.2356 ) between ssLFAvs npRT-PCR, sensitivity of $38.9 \%$ (exact $95 \% \mathrm{Cl}$ of $20.3 \%$ to $61.4 \%$ ), specificity of $89.4 \%$ (exact $95 \% \mathrm{Cl}$ of $84.1 \%$ to $93.1 \%$ ), PPV of $26.9 \%$ (95\% Cl of $13.7 \%$ to $46.1 \%$ ), NPV of $93.6 \%$ (exact $95 \% \mathrm{Cl}$ of $88.8 \%$ to $96.4 \%$ ), $\mathrm{LR}+$ of 3.67 (95\% Cl of $1.79-7.51$ ), LR - of $0.68(95 \% \mathrm{Cl}$ of $0.47-0.99)$.

Conclusion. Our data showed that snort-spit saliva RT-PCR testing had acceptable diagnostic performance characteristics and can potentially be used as an alternative to the standard nasopharyngeal/oropharyngeal swab RT-PCR test for COVID-19 in certain situations. However, our data also showed that snort-spit saliva antigen testing using lateral flow assay did not offer acceptable performance.

Key Words: SARS-CoV-2, reverse transcription polymerase chain reaction, saliva

\section{INTRODUCTION}

${ }^{*}$ Dr. Carrillo and Dr. Sarmiento share primary authorship for this manuscript.

Corresponding author: Ryner Jose D. Carrillo, MD, MSc (Clin Epid) University of the Philippines-Philippine General Hospital

College of Medicine

University of the Philippines-Manila

Taft Avenue, Ermita Manila 1000, Philippines

Email: rdcarrillo@up.edu.ph
In the era of COVID-19 or SARS-CoV-2 pandemic, determining infection and infectivity by viral RNA reverse transcription polymerase chain reaction (RT-PCR) has been the diagnostic method of choice. Studies have tested different samples- nasopharyngeal (NP) or oropharyngeal (OP) swabs, bronchial lavage, whole saliva, and deep throat saliva. The nasopharyngeal swab RT-PCR has been considered by the World Health Organization as the sample type of choice for diagnostics ${ }^{1}$. However, the collection of NP specimens can be regarded as invasive and causes discomfort 
resulting in coughing in most patients and increasing the risk of nosocomial spread of respiratory viruses. The process of obtaining these samples exposes the healthcare professional to infection such that the process will require a high level of personal protective equipment (PPE).

Salivary glands have been proven to be reservoirs for COVID-19 asymptomatic infection. ${ }^{2}$ Saliva or oral fluid can easily be obtained by asking the patient to cough out saliva from the throat to container sample for RT-PCR. ${ }^{3}$ Serial self-collected saliva testing yielded positive in $91.7 \%$ $(11 / 12)$ patients showing that a self-collected saliva is a viable option in detecting $2019-\mathrm{nCoV}{ }^{4}$ In a 2020 study by Wyllie, saliva and nasopharyngeal samples were compared, and they detected SARS-CoV-2 in saliva from two subjects who were negative by nasopharyngeal swabs. ${ }^{5}$ They also found that saliva yielded higher detection sensitivity and consistency throughout infection. A meta-analysis by Czumbel in 2020 found that sensitivity of saliva tests is high at 91\% (CI 80-99\%) while sensitivity for nasopharyngeal swab is at $98 \%$ (CI 89-100\%) among previously confirmed COVID-19 patients. $^{6}$

An affordable, easily deployable, rapid, and accurate self-collected test for COVID-19 may be a game-changer in the fight against this pandemic. Rapid antigen tests using lateral flow immunoassays have been explored as a possible alternative in settings where RT-PCR may not be readily available. One operational drawback is that these tests rely mostly on nasopharyngeal specimens. Thus, professional sampling and the use of personal protective equipment during sampling and processing are still necessary. Using more readily available and easily collected samples such as saliva may help decrease the need for protective equipment and decrease the risk of exposure to healthcare workers. Saliva being used in antigen tests may also be used in largescale and point-of-care settings. ${ }^{7}$

The BIOCREDIT COVID-19 Ag test (RapiGEN Inc., Gyeonggi-do, 14119, Korea) is a rapid antigen test already being used in the community. It has a published product description of having $94.4 \%$ sensitivity and $100 \%$ specificity in a population of 134 and a disease prevalence of $26.5 \% .{ }^{8}$ Table 1 shows the pooled results from European and South American subjects.

This study aimed to validate self-collected snorted and spit saliva in detecting COVID-19 using RT-PCR (ssRT-PCR) and lateral flow antigen test (ssLFA) versus nasopharyngeal swab RT-PCR (npRT-PCR).

Table 1. Biocredit Rapid antigen test product insert results

\begin{tabular}{lrcr} 
& + RT PCR & - RT PCR & Total \\
Biocredit COVID Ag + & 34 & 0 & 34 \\
Biocredit COVID Ag- & 2 & 134 & 136 \\
\hline Total & 36 & 134 & 136 \\
\hline
\end{tabular}

\section{METHODS}

This was a cross-sectional diagnostic accuracy study, with prospective recruitment, using the STARD classification". This research was conducted from September to November 2020 at the Philippine General Hospital (PGH). The study secured approval from the Institutional Biosafety and Biosecurity Committee and the University of the Philippines Manila Research Ethics Board before commencing (Registration: UPMREB 2020-301-01). Data collection was planned before the index test, and the reference standard was performed.

Two hundred eight (208) symptomatic adults (19-65 years old) suspected and probable cases of SARS-CoV-2 infections, using the WHO case definitions, were enrolled in the study. ${ }^{1}$ These included health care workers and individuals who consulted at the outpatient $\mathrm{s}$ wabbing station of the hospital. All were ambulatory. Those who were severely symptomatic, non-ambulatory, and requiring $\mathrm{O}_{2}$ support or intubation were excluded from the study.

\section{Specimen collection and transport}

A designated health care worker (HCW) supervised the snort-spit saliva sample collection while trained medical technologists obtained the nasopharyngeal swab sample according to standard operating procedures. The HCW instructed subjects to snort and then spit $1-2 \mathrm{~mL}$ of the whole saliva directly into two different sterile tubes: the first tube contains viral transport medium (VTM) to be processed for RT-PCR testing, and the second tube contains the assay diluent for the lateral flow immunoassay.

Following the snort-spit saliva collection, the medical technologist performed the nasopharyngeal swabbing on bilateral nostrils using one pledget. The pledget was then placed in the tube with VTM. Triple-packed VTMs of both snort-spit saliva and NP swabs were then immediately transported to the PGH Molecular Laboratory for processing.

\section{Rapid antigen testing of saliva specimens}

BIOCREDIT COVID-19 Ag test (RapiGEN Inc., Gyeonggi-do, 14119, Korea) was used for the rapid testing part. It was a lateral flow immunochromatographic assay intended for the qualitative detection of SARS-CoV-2 antigen. ${ }^{8}$ This assay was designed for use with nasopharyngeal swab samples but was used in this study on saliva samples. The HCW performed the rapid test immediately after saliva collection. Three to four drops of the obtained snortspit saliva and assay diluent mixture were squeezed into the test device's sample well. Results were considered positive if two bands appear, one red control line and one black test line, after a standard waiting time and regardless of color intensity. 


\section{Specimen processing for RT-PCR}

The NP swab and snort-spit saliva samples were aliquoted and heat-inactivated at 65 Celsius for 10 minutes or chemically inactivated using the lysis buffer before RNA extraction. SARS-CoV-2 viral RNA amplification and detection were performed following standard operating procedures. Viral RNA extraction was done using GenAmplify $^{\mathrm{TM}}$ Viral RNA Purification Kit (The Manila HealthTek, Inc.). SARS-CoV-2 RT-PCR detection was then performed using either Novel Coronavirus (2019nCoV) Nucleic Acid Diagnostic Kit (Sansure Biotech Inc.) or Real-Time Fluorescent RT-PCR Kit for Detecting SARS-CoV-2 (BGI Genomics Co. Ltd.). Given an NP swab and snort-saliva specimen pair, it was ensured that the same SARS-CoV-2 RT-PCR detection kit was used. All kits used were validated before use.

\section{RESULTS}

A total of 208 subjects were recruited and were able to give their informed consent to participate. However, two participants got swabbed first before snort-spit saliva collection, one participant had her NP RT-PCR processed using the automated process, and eight had invalid RT-PCR results. A total of 11 participants were excluded from the final set, and the final number was 197. Participants' median age was 32 years (range 22-64), and 123 participants (62.4\%) were female. The prevalence of COVID-19 infection in the study cohort, based on the reference method of npRT-PCR, was 9\% (18/197, 95\% CI of 5.5\% - 14.1\%). The snort-spit saliva RT PCR test (ssRT-PCR) and snort-spit antigen test (ssLFA), however, showed higher positivity rates, both $13.1 \%$ (26/197), compared to npRT-PCR.

The ssRT-PCR showed a substantial agreement of 92.89\% (95\% exact CI of $88.4 \%$ to $96.1 \%$; Cohen's Kappa of 0.6435 ) with npRT-PCR (see Table 2). The ssRT-PCR has a positive likelihood ratio of 13.66 and a negative likelihood ratio of 0.178 . This means that if the pretest probability of a case is $9 \%$, and the ssRT-PCR result is positive, the post-

Table 2. Snort-spit saliva RT-PCR vs. Nasopharyngeal swab RT-PCR for SARS-Cov-2

\begin{tabular}{lccc} 
& npRT-PCR + & npRT-PCR - & Total \\
SSRT-PCR + & 15 & 11 & 26 \\
SSRT-PCR - & 3 & 168 & 171 \\
\hline Total & 18 & 179 & 197 \\
\hline
\end{tabular}

Table3. Snort-spit saliva antigen test vs. Nasopharyngeal swab RT-PCR for SARS-Cov-2

\begin{tabular}{lccc} 
& npRT-PCR + & npRT-PCR - & Total \\
ssLFA + & 7 & 19 & 26 \\
ssLFA - & 11 & 160 & 171 \\
\hline Total & 18 & 170 & 197 \\
\hline
\end{tabular}

test probability will be $57.7 \%$. If the pre-test probability is $9 \%$, and the ssRT-PCR result is negative, the post-test probability will be $1.7 \%$. The sensitivity is $83.3 \%$ (95\% exact CI of $60.8 \%$ to $94.2 \%)$ and the specificity is $93.9 \%$ (95\% exact CI of $89.3 \%$ to $96.5 \%$ ).

The ssLFA showed an only fair agreement of $84.77 \%$ (95\% exact CI of 79.1\% to 89.5\%; Cohen's Kappa of 0.2356 ) with npRT-PCR (see Table 3). The ssLFA has a positive likelihood ratio of 3.67 and a negative likelihood ratio of 0.68 . This means that if the pretest probability of a case is $9 \%$, and the ssLFA result is positive, the post-test probability will be $26.6 \%$. If the pre-test probability is $9 \%$, and the ssLFA result is negative, the post-test probability will be $6.3 \%$.

Further analysis of the data (Figure 1) shows considerable clustering of ssRT-PCR cycle threshold (Ct-values) towards the higher $\mathrm{Ct}$-values in samples read as negative by the reference standard (npRT-PCR), suggesting some form of systematic error.

Comparison of the distribution of npRT-PCR and ssRT-PCR Ct-values with positive and negative ssLFA results shows that ssLFA misclassified a considerable proportion of cases with detectable viral RNA by RTPCR in both the npRT-PCR and ssRT-PCR and that the misclassification error is random as it does not demonstrate any pattern across the range of $\mathrm{Ct}$-values (number of PCR cycles to achieve the desired signal). (Figure $2 \mathrm{~A}$ and $\mathrm{B}$.)

\section{DISCUSSION}

Nasopharyngeal swab RT PCR is currently considered the diagnostic method of choice for SARS COV 2 testing by the WHO guidelines. ${ }^{1}$ Due to its invasive technique, high risk for coughing, increased risk of exposure to HCWs, and higher demand for protective equipment for sample

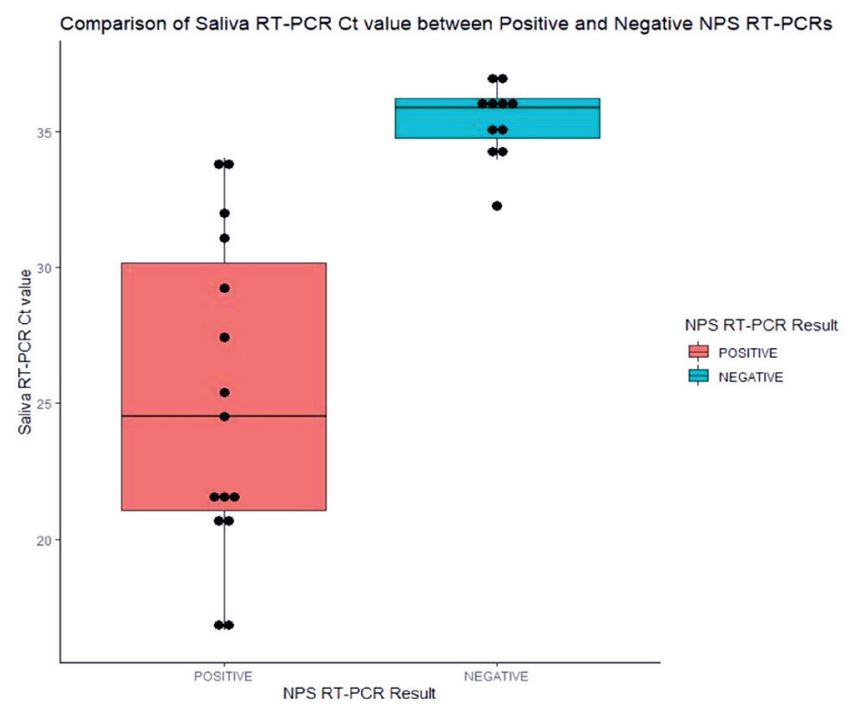

Figure 1. Comparison of Saliva RT-PCR Ct values between Positive and Negative NPS RT-PCRs. 

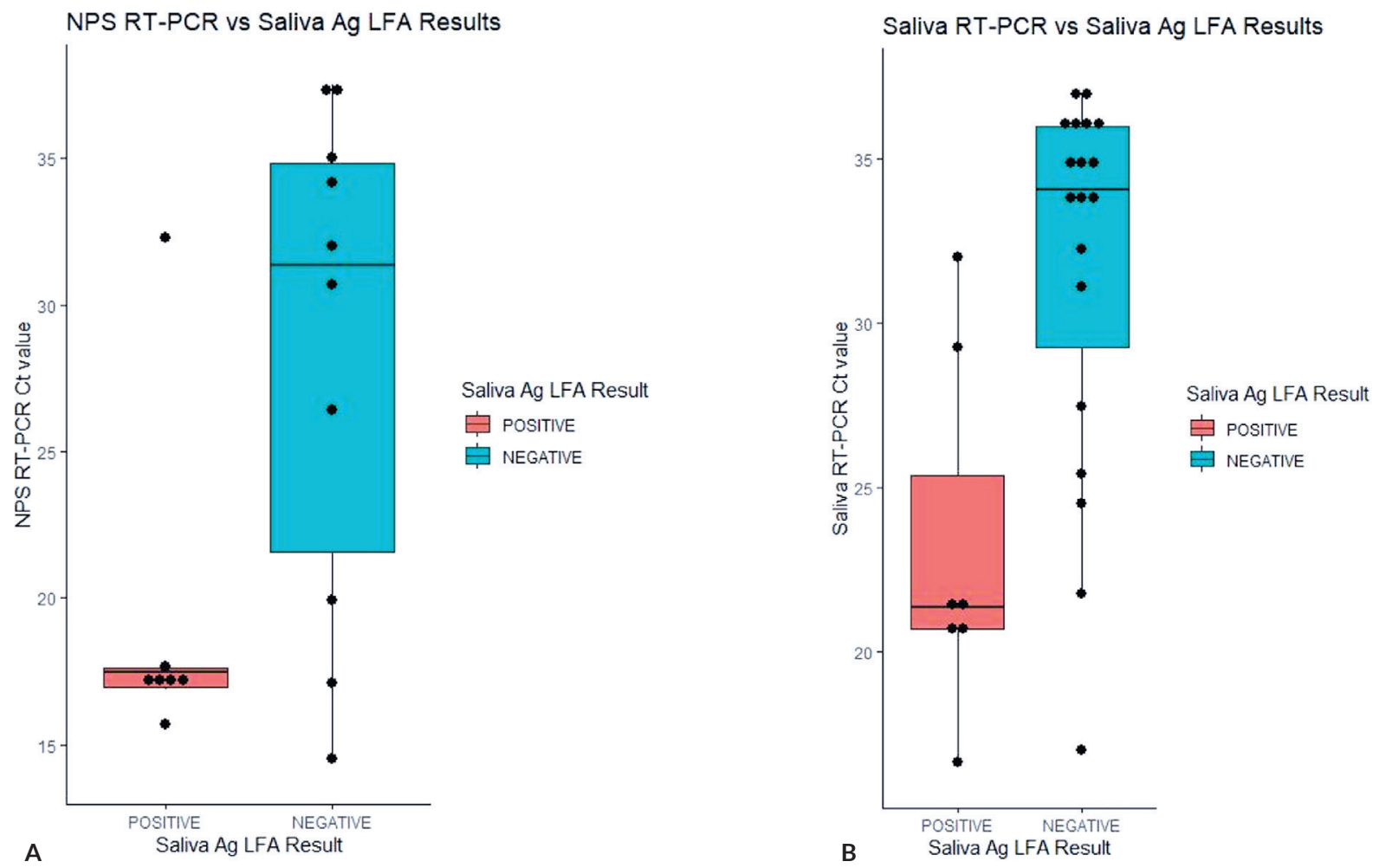

Figure 2. Comparison of snort-spit saliva rapid antigen testing (ssLFA) with (A) snort-spit saliva RT-PCR (ssRT-PCR) and (B) nasopharyngeal RT-PCR (npRT-PCR).

collection, several studies have explored saliva- or sputumbased testing. The current study's difference is that it utilizes the snort-spit technique of collection, aiming to sample both the nasopharyngeal mucous and whole saliva.

\section{Snort-spit Whole Saliva RT-PCR}

Using the npRT PCR as the reference standard, the diagnostic sensitivity and specificity of ssRT-PCR in detecting COVID19 infection is high at $83.3 \%$ and $93.9 \%$, respectively. This is consistent with previous cross-sectional studies by Pasomsub (2020), with saliva having sensitivity and specificity of $84.2 \%$ and $98.9 \%$, respectively, and a more recent study by Altawalah (2020) showing sensitivity and specificity of $83.43 \%$ (95\% CI: 79.07-87.20) and 96.71 \% (95 \% CI: 94.85-98.04 \%), respectively. ${ }^{10,11}$ However, the presence of a wide variation in the assay performance based on several more studies results, the WHO does not yet recommend using saliva as the sole specimen for routine clinical diagnostics. ${ }^{1}$

The positive and negative likelihood ratio estimates in this study for ssRT-PCR suggest usefulness in an actual clinical setting. For example, in a patient assigned a pretest probability of $50 \%$, that is, the clinical decision-maker is at an equipoise, a positive ssRT-PCR can increase the post-test probability to $93.2 \%$, and a negative ssRT-PCR can decrease the post-test probability to $15.1 \%$, enough to be used as the basis for clinical decisions.
The current study provides some evidence that the detection rate of SARS-COV-2 could be higher in snortspit samples than nasopharyngeal swabs $(13.3 \%$ vs. $9 \%$ positivity rate, respectively). If cross-contamination at any point in the processing can be ruled out, a higher positive test rate will suggest an actual increase in detection rate. Furthermore, because there was considerable clustering of the Ct-values towards the higher end of the assay range, in the group of positive samples by ssRT-PCR but negative by npRT-PCR, the npRT-PCR assay could be systematically misclassifying those sample with very low viral copies. Thus it is very tempting to consider the possibility that ssRTPCR could, in reality, be more sensitive than npRT-PCR in SARS-CoV-2 detection. If indeed this could be true, it would be easy to see that such will be the case as samples collected by snorting and spitting collects fluid from a larger area, including the nasal, nasopharyngeal, oropharyngeal, and oral cavities, and the total fluid collected is far larger in volume compared to nasopharyngeal and oropharyngeal swabbing. Further investigation using carefully designed experiments is suggested to explore this assertion. The preamplification extraction method and validation of SARSCoV-2 RT PCR interpretation criteria specifically for snortspit samples may be pursued as a future direction to provide additional useful information to strengthen the evidence for the potential of snort-spit as an alternative sample for SARS-CoV-2 detection. 


\section{Snort-spit Saliva Rapid Antigen Assay (Lateral Flow Assay)}

We explored the possibility of snorting and spitting mucus and saliva to a collection bottle and testing it on a lateral flow device for rapid antigen detection using the BIOCREDIT COVID-19 Ag test (RapiGEN Inc., Gyeonggi-do, 14119, Korea). With a snort-spit sample, there was only fair agreement and poor sensitivity and specificity when using antigen than what is published in the product insert, which uses nasopharyngeal swab. The current study employed a modified sample processing method to perform the antigen test, which may be a limitation. Hence, the utility of snort-spit saliva samples using rapid antigen detection may be further explored in the future when the prevalence of the disease is higher and when further validation can be done. Strategizing maneuvers, like spaced repeated selftesting, can be done in future studies considering the existing burden of disease.

\section{CONCLUSION}

Snort-spit saliva RT-PCR can be an acceptable alternative to nasopharyngeal RT-PCR, especially in a setting where nasopharyngeal specimen cannot be obtained. There is some evidence to suggest that snort-spit saliva RTPCR may be a more sensitive method than nasopharyngeal RT-PCR. Snort-spit rapid antigen testing is not an acceptable alternative to nasopharyngeal RT-PCR. Further investigation into the superiority of snort-spit saliva RTPCR over nasopharyngeal RT-PCR is recommended.

\section{Statement of Authorship}

All authors participated in the data collection and analysis and approved the final version submitted.

\section{Author Disclosure}

All authors declared no conflicts of interest.

\section{Funding Source}

This paper was funded by the UP-PGH 2020 COVID Research Grant for Medical Specialist/Consultants.

\section{REFERENCES}

1. World Health Organization. Diagnostic testing for SARS-CoV-2. Interim guidance [Internet]. 2020 [cited 2021 Jan]. Available from: https://www.who.int/publications/i/item/diagnostic-testing-for-sarscov-2

2. Xu J, Li Y, Gan F, Du Y, Yao Y. Salivary Glands: Potential Reservoirs for COVID-19 Asymptomatic Infection. J Dent Res. 2020 Jul;99(8):989. doi: 10.1177/0022034520918518. Epub 2020 Apr 9. PMID: 32271653.

3. Khurshid Z, Asiri FYI, Al Wadaani H. Human Saliva: NonInvasive Fluid for Detecting Novel Coronavirus (2019-nCoV). Int J Environ Res Public Health. 2020 Mar 26;17(7):2225. doi: 10.3390/ ijerph17072225. PMID: 32224986; PMCID: PMC7178089.

4. To KK, Tsang OT, Yip CC, Chan KH, Wu TC, Chan JM, et al. Consistent Detection of 2019 Novel Coronavirus in Saliva. Clin Infect Dis. 2020 Jul 28;71(15):841-843. doi: 10.1093/cid/ciaa149. PMID: 32047895; PMCID: PMC7108139.

5. Wyllie AL, Fournier J, Casanovas-Massana A, Campbell M, Tokuyama M, Vijayakumar P, et al. Saliva or Nasopharyngeal Swab Specimens for Detection of SARS-CoV-2. N Engl J Med. 2020 Sep 24;383(13):1283-1286. doi: 10.1056/NEJMc2016359. Epub 2020 Aug 28. PMID: 32857487; PMCID: PMC7484747.

6. Czumbel LM, Kiss S, Farkas N, Mandel I, Hegyi A, Nagy Á, et al. Saliva as a Candidate for COVID-19 Diagnostic Testing: A MetaAnalysis. Front Med (Lausanne). 2020 Aug 4;7:465. doi: 10.3389/ fmed.2020.00465. PMID: 32903849; PMCID: PMC7438940.

7. European Centre for Disease Prevention and Control. Options for the use of rapid antigen tests for COVID-19 in the EU/EEA and the UK [Internet]. 2020 [cited 2021 Jan]. Available from:https://www. ecdc.europa.eu/en/publications-data/options-use-rapid-antigen-testscovid-19-eueea-and-uk

8. RapiGen. BIOCREDIT COVID-19 Ag. RapiGen [Internet]. 2020 [cited 2021 Jan]. Available from: https://www.biovendor. com/file/13419/1-2\%20IFU_Covid-19_Ag_Antigen_Multi\%20 Language\%20SEP2020Watermarked.pdf?version $=202009300841$

9. Bossuyt, P, Reitsma, J, Bruns, D, Gatsonis, C, Glasziou, P, Irwig, L, et al., J. STARD 2015: An updated list of essential items for reporting diagnostic accuracy studies. BMJ. 2015 Oct; h5527. https://doi. org/10.1136/bmj.h5527

10. Pasomsub E, Watcharananan SP, Boonyawat K, Janchompoo P, Wongtabtim G, Suksuwan W, et al. Saliva sample as a non-invasive specimen for the diagnosis of coronavirus disease 2019: a crosssectional study. Clin Microbiol Infect. 2020 May 15:S1198-743X (20)30278-0. doi: 10.1016/j.cmi.2020.05.001. Epub ahead of print. PMID: 32422408; PMCID: PMC7227531.

11. Altawalah H, AlHuraish F, Alkandari WA, Ezzikouri S. Saliva specimens for detection of severe acute respiratory syndrome coronavirus 2 in Kuwait: A cross-sectional study. J Clin Virol. 2020 Nov;132:104652. doi: 10.1016/j.jcv.2020.104652. Epub 2020 Oct 1. PMID: 33053493; PMCID: PMC7527795. 